\title{
Clear cell chondrosarcoma with secondary aneurysmal bone cyst changes
}

Timothy $\underline{T a y}^{1}$, MBBs, Steven Bak Siew Wong $^{2}$, MBChB, MMed, Kesavan s/o Sittampalam ${ }^{1}$, MBBS, FRCPA, Denny Tjiauw Tjoen $\underline{\text { Lie }}^{3}$, MBBS, FRCS

\begin{abstract}
Clear cell chondrosarcoma is a rare cartilaginous tumour of low-grade malignancy. Although it has a characteristic histological appearance, its radiological features and clinical presentation often mimic a benign lesion. Herein, we describe the case of a patient with a clear cell chondrosarcoma of the right proximal femur that had an atypical appearance of chronic avascular necrosis on initial plain radiographs, which made preoperative diagnosis a challenge. In addition, the tumour also had extensive areas of aneurysmal bone cyst-like changes, which is not only a rare histologic phenomenon in clear cell chondrosarcoma, but also a confounding factor in the interpretation of the radiologic findings.
\end{abstract}

Keywords: aneurysmal, bone cyst, chondrosarcoma, clear cell

\section{INTRODUCTION}

Clear cell chondrosarcoma is a rare low-grade malignant tumour that was first described by Unni et al in 1976. ${ }^{(1)}$ Since its first description, the clinical, radiological and histological features of clear cell chondrosarcoma have become increasingly well defined. However, this entity can still present with diagnostic difficulties, as its radiological features and somewhat indolent clinical behaviour in the early course of the illness often mimics a benign lesion. Awareness of this entity is important to avoid misdiagnosis, as early and adequate removal of this malignancy with clear margins has been shown to be curative in most cases; delayed diagnosis and inadequate removal by curettage, however, leads to a higher risk of local recurrences, metastases and death. ${ }^{(1-2)}$ Herein, we describe the case of a patient with a clear cell chondrosarcoma of the right proximal femur, with an atypical clinical presentation of avascular necrosis and secondary osteoarthritis of the right hip, associated with a histologic component of extensive aneurysmal bone cyst-like areas, which is a rare phenomenon in clear cell chondrosarcomas.

\section{CASE REPORT}

A 32-year-old Chinese woman presented with right hip pain that had persisted for five years. The pain had progressively worsened over the past six months. It was mechanical in nature, occurring during jogs and associated with a limited range of movement of her right hip. She had no history of chronic alcoholism, steroid use or previous trauma to her right hip. She was ambulatory without any physical aid.

Physical examination revealed a decreased range of motion on internal and external rotation, as well as adduction of the right hip joint. Her right lower limb was also observed to be shortened by $1 \mathrm{~cm}$ when compared to the left left lower limb.

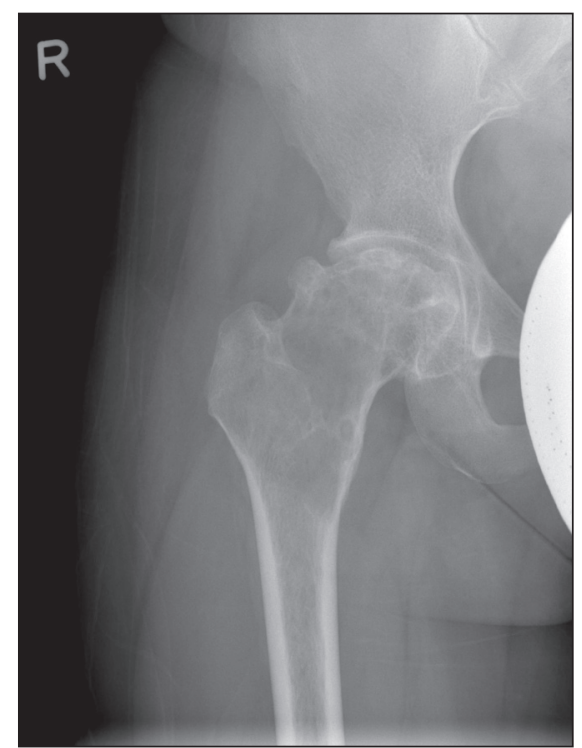

Fig. 1 Anteroposterior plain radiograph of the right hip joint shows marked deformity and flattening of the articular portion of the right femoral head. The articular surface irregularity and subchondral cystic changes seen mimic the appearance of avascular necrosis. Note the mildly expanded appearance of the femoral neck with cortical thinning and the homogeneous lucent appearance extending to the subtrochanteric zone, which were detected only on the second review. The relative absence of corresponding degenerative changes at the right acetabulum is incongruous with the changes in the femoral head.

Her gait was noted to be stiff and antalgic. Initial plain radiography of her right hip joint (Fig. 1) showed deformity of the right femoral head with articular surface irregularity and subchondral cystic changes, accompanied by mild inferomedial joint space narrowing. The subchondral cysts varied in size and opacity. These radiographic features were reported as consistent with chronic avascular necrosis. At this time, a homogeneous lucent area with slightly ill-defined but smooth margins at the femoral neck and lesser trochanteric region was not appreciated.

${ }^{1}$ Department of Pathology, ${ }^{2}$ Department of Diagnostic Radiology, ${ }^{3}$ Department of Orthopaedic Surgery, Singapore General Hospital, Singapore Correspondence: Dr Timothy Tay, Resident, Department of Pathology, Singapore General Hospital, 20 College Road, Level 10, Diagnostics Tower, The Academia, Singapore 169856. timothy.tay@mohh.com.sg 


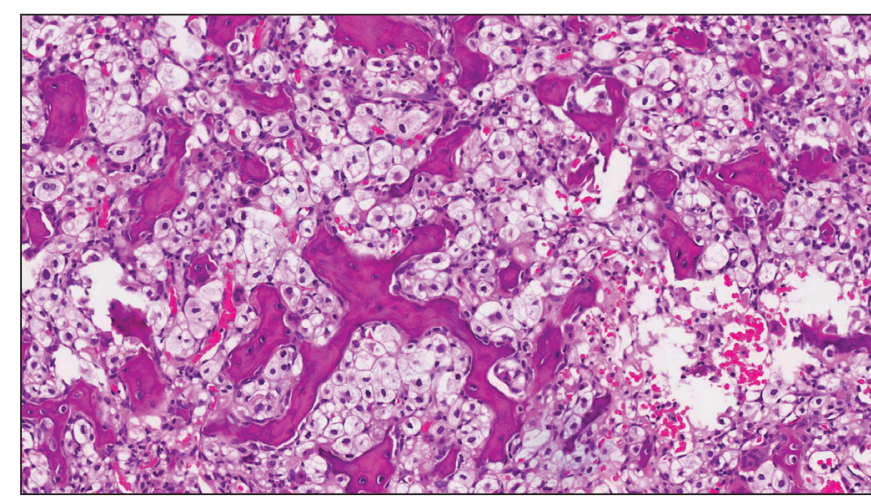

Fig. 2 Photomicrograph shows characteristic tumour cells with clear cytoplasm, well-defined cytoplasmic borders, and central, round-to-oval nuclei with woven bone scattered in between the cells (Haematoxylin \& eosin, $\times 200)$.

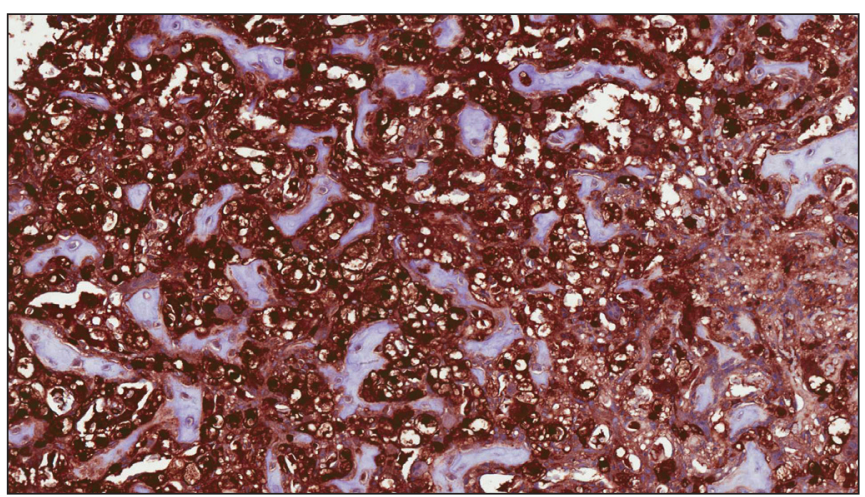

Fig. 3 Photomicrograph shows tumour cells that are positive for S100 (S100 immunohistochemical stain, $\times 200$ ).

Furthermore, the acetabulum showed no marginal osteophytes or erosion commensurate with osteoarthritis.

The patient underwent a right total hip replacement. Intraoperatively, the surgeon noted that the proximal femur had cavities that were filled with a gritty-textured lesion. This lesion was curetted and sent for histologic examination. Postoperatively, the patient had an uneventful recovery.

Histological examination of the bone curettings showed the presence of aggregates of clear cells with intervening thin spicules of sclerotic, woven bone (Fig. 2). These tumour cells had well-defined cytoplasmic borders, clear cytoplasm, and a central, round-to-oval nuclei with vesicular chromatin. Mitoses were rare. Focal conventional chondroid matrix was also observed. Multinucleated giant cells were seen at the periphery of the tumour. The tumour cells immunostained positively for S100 (Fig. 3) and negatively for cytokeratins MNF-116 and CAM5.2. Areas of aneurysmal bone cyst-like changes composed of multiloculated cystic spaces containing blood and old haemorrhage, with a fibrous wall devoid of lining epithelium (Fig. 4), were also conspicuous. Reactive bone formation was also present within the fibrous septae. These features are in keeping with a clear cell chondrosarcoma with areas of aneurysmal bone cyst-like changes.

Positron emission tomography/computed tomography of the patient's whole body was performed on follow-up. Areas of radioactivity were demonstrated at the arthroplasty site and

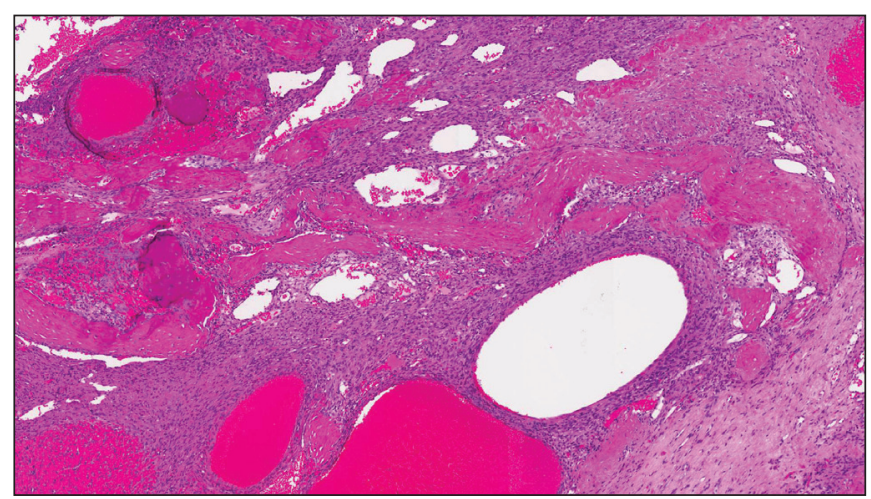

Fig. 4 Photomicrograph shows areas of the tumour with aneurysmal bone cyst-like changes composed of multiloculated cystic spaces containing blood, with a fibrous wall devoid of lining epithelium (Haematoxylin \& eosin, $\times 100)$.

in the surrounding soft tissue, but these were deemed to be postsurgical in nature. No 16-fluorodeoxyglucose-avid lesions were seen.

\section{DISCUSSION}

Clear cell chondrosarcoma is a rare tumour, accounting for only $2 \%-4 \%$ of chondrosarcomas. ${ }^{(3)}$ It usually occurs in the third to fifth decade of life, although the age of presentation varies widely (from the second to the seventh decade of life). ${ }^{(1)}$ It is more common in men than women, and the symptoms at presentation can be nonspecific. Some are asymptomatic, with the tumour detected incidentally on imaging, while others are associated with pain that ranges from 1 year to more than 5 years in duration, probably due to the slow-growing nature of the tumour. Some patients also present with a pathological fracture. ${ }^{(1,2,4)}$

Although most of these tumours occur at the epiphyseal equivalent regions of long bones (e.g. the femur, humerus and tibia), rare sites such as the skull, spine, pelvis, phalanges, and even the larynx have been described..$^{(1,2,4-7)}$ These tumours usually appear as expansile, radiolucent lesions centred in the epiphysis (with or without extension to the metaphysis) on radiography. ${ }^{(1)}$ The margins of these tumours are usually well defined, although they can be less defined in larger lesions. The cortex is usually intact and periosteal new bone formation is usually absent. Areas of calcification can sometimes be seen within the lesion. These features can easily lead to an incorrect diagnosis of a benign lesion, especially when the slow-growing lesion does not show significant interval growth on serial radiographs. It can also be difficult to distinguish clear cell chondrosarcoma from chondroblastoma due to their similar radiographic features and common epiphyseal location. In rare cases of dedifferentiated clear cell chondrosarcoma, the tumour will have a bimorphic pattern with a low-grade lesion juxtaposed against an area with marked destruction of the bone and poorly defined margins. ${ }^{(8)}$ Other differential diagnoses on radiography include giant cell tumour and aneurysmal bone cyst. 
Aneurysmal bone cyst-like components were detected in our patient. Secondary aneurysmal bone cysts are known to be associated with underlying bone malignancies and fibrous dysplasia. The radiographic features of the aneurysmal bone cyst depend on the phase at presentation. It is seen as illdefined osteolysis in the initial phase and as locally aggressive expansions and destructions, with marked periosteal reaction, in the growth phase. Progressive rim ossification and internal calcification are seen in the stabilisation phase.

Magnetic resonance (MR) imaging may demonstrate the underlying bone malignancy. ${ }^{(9)}$ It can also be used to examine the extent of osseous and extraosseous involvement. Clear cell chondrosarcoma shows low to intermediate signal intensity on T1-weighted MR imaging, heterogeneous high signal intensity on T2-weighted MR imaging and prominent enhancement with the use of intravenous contrast medium.

Histologically, clear cell chondrosarcoma has a characteristic appearance. It consists of lobules or sheets of cells with clear cytoplasm, well-defined cytoplasmic borders, and central, round-to-oval nuclei with vesicular chromatin. Benign osteoclastlike giant cells may also be seen at the periphery. Fine lines of calcification, or osteoid or bone formation can be found scattered among the tumour cells. Chondroid matrix may also be present. ${ }^{(1,10)}$ In some cases, areas of conventional chondrosarcoma may also be seen, prompting some to argue that clear cell chondrosarcoma is a variant derived from conventional chondrosarcoma, rather than from a chondroblastoma that has undergone malignant change.(1) Other features that may be present include areas of aneurysmal bone cyst-like changes, ${ }^{(4)}$ which was seen in the present case.

The differential diagnoses of clear cell chondrosarcoma include chondroblastoma, osteoblastoma, epiphyseal osteosarcoma and chondroblastic osteosarcoma (especially if the tumour is located in the head and neck region), giant cell tumour and metastatic renal cell carcinoma. Due to the characteristic histological features of clear cell chondrosarcoma, a correct diagnosis is reached in most cases. Although chondroblastoma shares certain features with clear cell chondrosarcoma (e.g. fine lines of calcification among the tumour cells, the presence of giant cells and a lobulated appearance), the tumour cells of chondroblastomas usually have a slightly acidophilic cytoplasm and nuclei that are oval to spindle-shaped with grooves. Cells with clear cytoplasm are also usually not present in chondroblastomas. Epiphyseal osteosarcoma and chondroblastic osteosarcoma usually have severe nuclear pleomorphism and atypical mitoses - two features that are not seen in clear cell chondrosarcoma. Clear cells are also not seen in osteoblastoma and giant cell tumours. Differentiation from a metastatic renal cell carcinoma can be aided via correlation with the patient's clinical history and/or immunohistochemistry. Clear cell chondrosarcomas are usually positive for S100 and type II collagen, and negative for cytokeratins, while the converse is true for renal cell carcinomas. ${ }^{(11)}$

Previous studies have shown that complete resection of the tumour with wide margins provides the best result (i.e. a lower risk of local recurrence, metastases and death). ${ }^{(1,2,4)}$ However, due to the slow-growing nature of the tumour and its often benign appearance on radiographs, it can be misdiagnosed as a benign lesion and treated with curettage or incomplete excision, leading to a high risk of local recurrence and metastatic disease. The common sites of metastasis include the lung and parts of the skeleton, such as the skull. ${ }^{(1,2,4,8)}$

In summary, clear cell chondrosarcoma is a rare cartilaginous tumour of low-grade malignancy. While adequate treatment with wide resection usually achieves an excellent prognosis for the patient, the tumour often mimics a benign lesion both clinically and radiographically, which could lead to an incorrect diagnosis and inadequate treatment. We herein report a case of clear cell chondrosarcoma that presented with atypical radiological features and extensive aneurysmal bone cyst-like changes, which made preoperative diagnosis a challenge. To avoid misdiagnosis and inadequate treatment, it is important that clinicians have an awareness of such an entity when presented with patients with bone lesions.

\section{REFERENCES}

1. Unni KK, Dahlin DC, Beabout JW, Sim FH. Chondrosarcoma: clear-cell variant. A report of sixteen cases. J Bone Joint Surg Am 1976; 58:676-83.

2. Ayoub KS, Grimer RJ, Carter SR, et al. Clear cell chondrosarcoma of bone. Sarcoma 1999; 3:115-9.

3. Greenspan A. Tumours of cartilage origin. Orthop Clin North Am 1989; 20:347-66.

4. Bjornsson J, Unni KK, Dahlin DC, Beabout JW, Sim FH. Clear cell chondrosarcoma of bone. Observations in 47 cases. Am J Surg Pathol 1984; 8:223-30

5. Kleist B, Poetsch M, Lang C, et al. Clear cell chondrosarcoma of the larynx: a case report of a rare histologic variant in an uncommon localization. Am J Surg Pathol 2002; 26:386-92.

6. Mokhtari S, Mirafsharieh A. Clear cell chondrosarcoma of the head and neck. Head Neck Oncol 2012; 4:13.

7. Hsu W, McCarthy E, Gokaslan ZL, Wolinsky JP. Clear-cell chondrosarcoma of the lumbar spine: case report and review of the literature. Neurosurgery 2011; 68:E1160-4

8. Kalil RK, Inwards CY, Unni KK, et al. Dedifferentiated clear cell chondrosarcoma. Am J Surg Pathol 2000; 24:1079-86.

9. Motamedi K, Seeger LL. Benign bone tumours. Radiol Clin North Am 2011; 49:1115-34.

10. Singh S, Ng KC, Tan SK. Clear cell chondrosarcoma - a report of 2 cases. Singapore Med J 1991; 32:154-7.

11. Masui F, Ushigome S, Fujii K. Clear cell chondrosarcoma: a pathological and immunohistochemical study. Histopathology 1999; 34:447-52. 